\title{
Cummins Marine Diesel Engine Wear Analysis Based on the Oil Ferrography Analysis Technology Xuewei Huang ${ }^{1, a^{*}}$, Suning $\mathrm{Bao}^{2}$, Huayu Dong ${ }^{3}$, Jin $\mathrm{Xu}^{4}$ \\ ${ }^{1}$ Zhenjiang Watercraft College, Jiangsu, China \\ ${ }^{2}$ Zhenjiang Watercraft College, Jiangsu, China \\ ${ }^{3}$ Zhenjiang Watercraft College, Jiangsu, China \\ ${ }^{4}$ Zhenjiang Watercraft College, Jiangsu, China \\ ahxwdyl@126.com
}

Keywords: Oil ferrography analysis; Wear; Cummins diesel engine;

Abstract. Most of marine diesel engine faults is caused by the wear. So, wear analysis is particularly important. In this paper, we have got the wear state and wear mechanism of the marine Cummins diesel engine by analyzing the selection of two typical oil sample with the oil ferrography analysis technology, and this paper also provided a certain theoretical basis for the next step maintenance decision-making.

\section{Introduction}

A large amount of data show that about $80 \%$ of the marine diesel engine fault eliminating human factors is caused by the wear ${ }^{[1]}$. So, the diesel engine wear analysis play an important role to ensure the normal operation of the diesel engine. The oil in diesel engine lubrication system have rich information, which about the friction pair of diesel engine, of the wear condition ${ }^{[2,3]}$. The potential hidden faults of the diesel engine can be early detected by analyzing the wear particles morphology, size, color, etc, which in the ship diesel engine lubricating oil using the oil and liquid iron spectrum analysis technique ${ }^{[4]}$. So the oil and liquid iron spectrum analysis technique is of great significance to optimize the maintenance plan, reduce the maintenance cost and the total cost of ownership.

\section{Experimental material and method}

\section{Experimental material}

\section{The oil sample I}

The oil sample of NTA855-M350 type Cummins marine diesel engine which on good condition.

\section{The oil sample II}

The oil sample of NTA855-M350 type Cummins marine diesel engine which is close to the overhaul period

\section{Experimental method}

\section{Sampling}

Extracting Iand II oil sample in the place which is of 0.5 times the oil depth under the oil surface by using the special sampler as soon as the machine stop working.

\section{Ferrogram making}

Making the ferrogram with the VIC-T type analytical ferrograph according to the steps.

Ferrogram analyzing

Placing the ferrogram on the ferrography microscope stage, and analysising the ferrogram with

Daheng-image image analysis system. 


\section{The results and discussion}

\section{The oil sample I}

As shown in figure.1, we getting the typical images by selecting 10 times ferrographic microscope objective lens magnification.

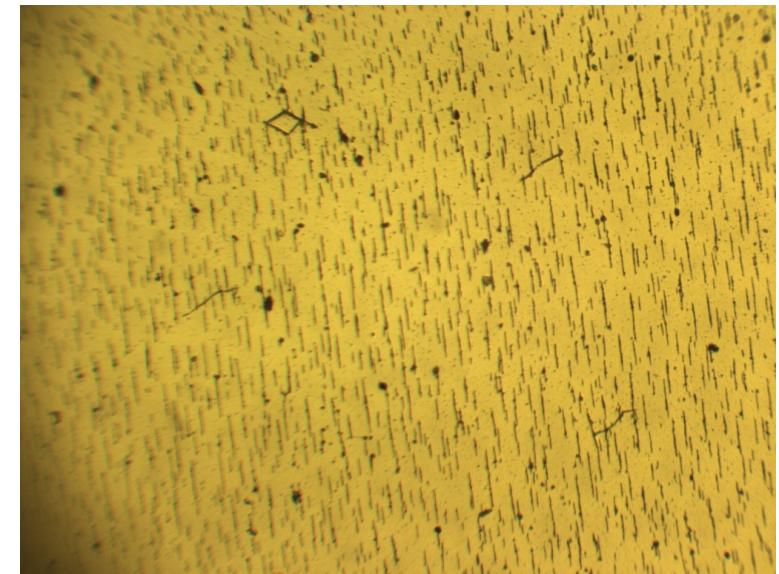

Figure.1 The ferrogram of oil sample $\square$ (10 times objective)

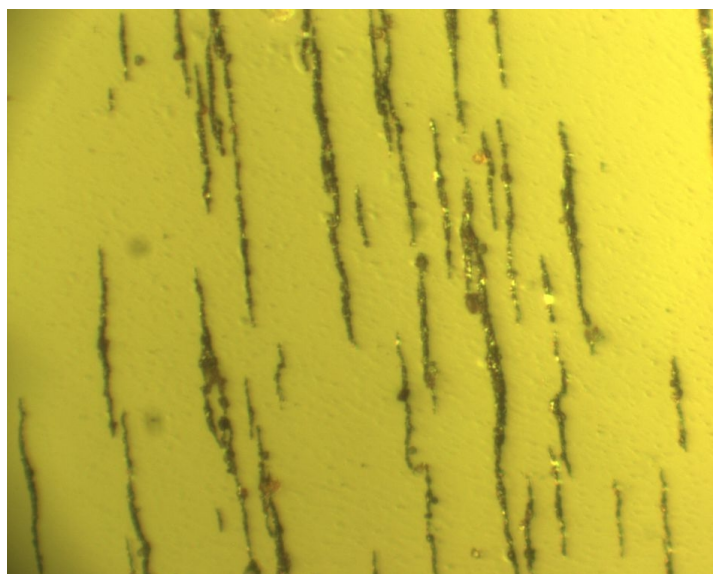

Figure.2 The ferrogram of oil samplel $\quad(50$

As shown in figure.1, we can learn that most of the particles are normal worn pellets, and the pellets size range is $1-13 \mu \mathrm{m}$, from which we could get the conclusion preliminary that the machine was in normal wear situation. However, we should make a further observation by magnifying these pellets.

Adjust object glass to 50 times than usual just as shown in figure.2. Then we can see clearly that the worn pellets are in the shape of scale, polished in surface, which is typical characteristic of normal worn pellets ${ }^{[5]}$. Thus we can say they are normally worn pellets. And a very few of pellets are in the shape of ball, but their diameter length is under $1 \mu \mathrm{m}$, which are typical characteristics of normal wear stage worn pellets.

\section{The oil sample II}

Adjust 10 times ferrographic microscope objective lens magnification than usual, then we can get the typical picture as shown in figure.3.

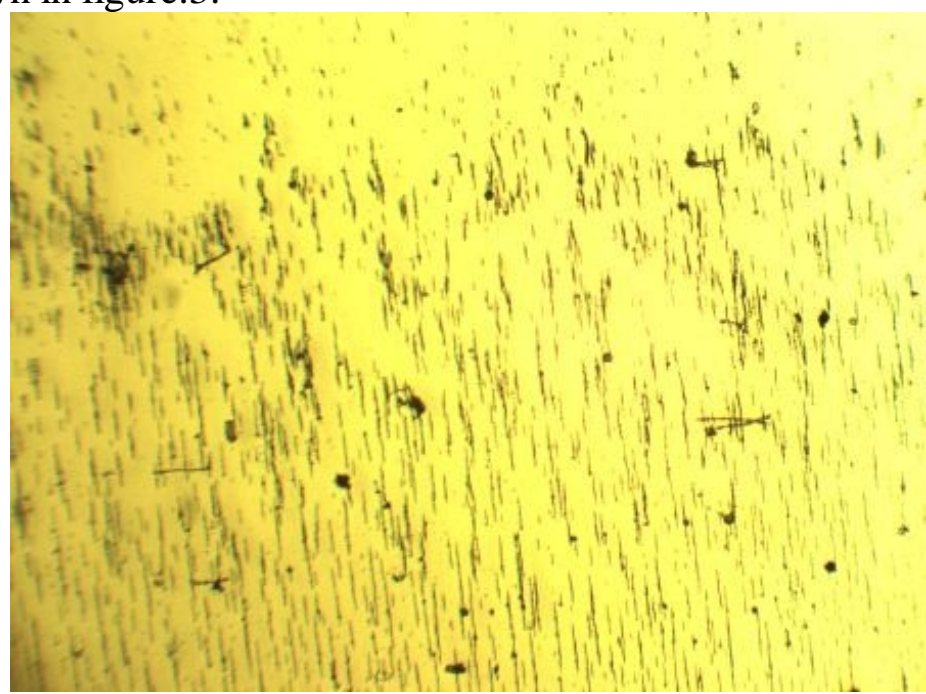

Figure. 3 The ferrogram of oil samplell(10 times objective)

As shown in figure. 3, we can learn that the worn pellets contain a significant amount of irregular shape pellets, which are abnormal worn pellets. Adjust 50 times ferrographic microscope objective lens magnification than usual, and then adjust the ferrogram, we got the image in figure.4, 5, 6, 7, 8 . 


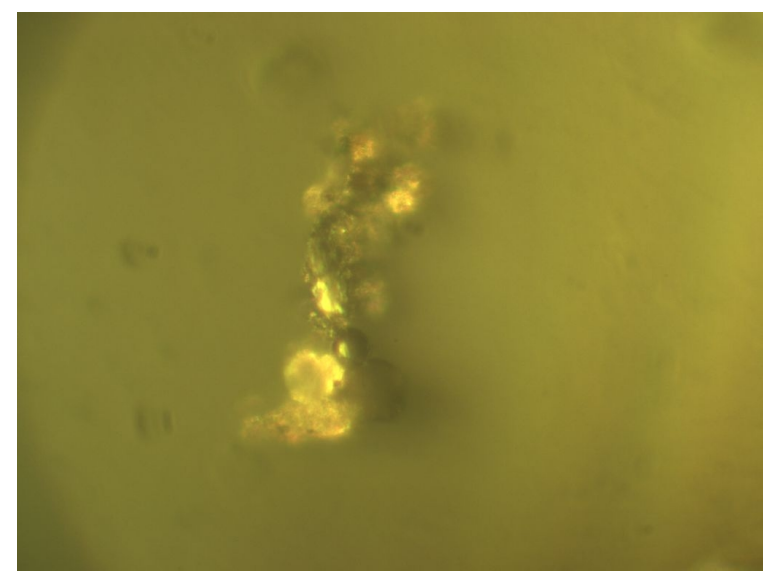

Fig.4 Spherical particles(50 times objective)

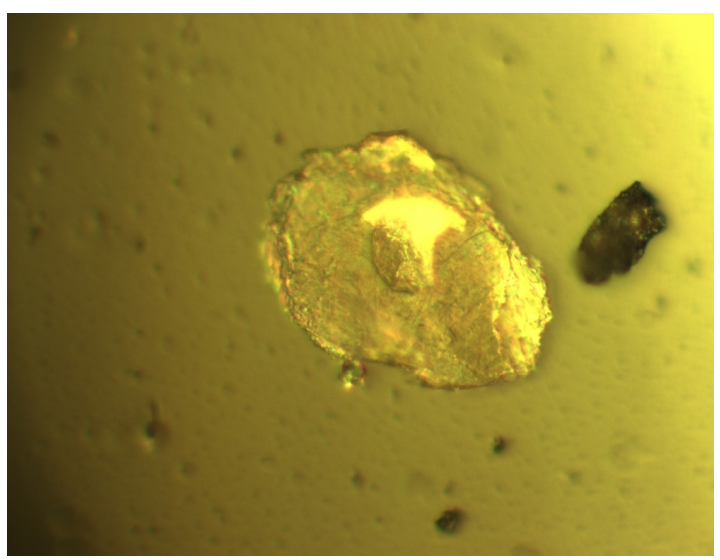

Fig.5 Sheet-shape grains(50 times objective)

As shown in figure. 4, we can see clearly that the worn pellets which has smooth surface is in the shape of ball, and the pellets diameter is $1.5 \mu \mathrm{m}$, which is typical characteristic of the initial stage fatigue wear worn pellets .

As shown in figure. 5, we can see clearly that the pellets which has smooth surface is irregular. The pellets length and the width is $12 \mu \mathrm{m}$ and $13 \mu \mathrm{m}$ respectively, which is typical characteristic of the initial stage fatigue wear worn pellets.

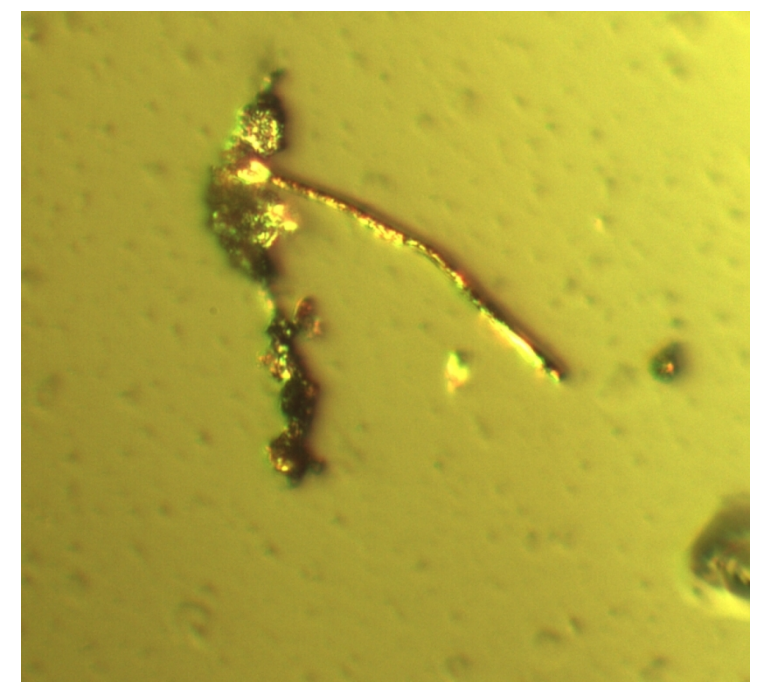

Fig.6 Cutting particles

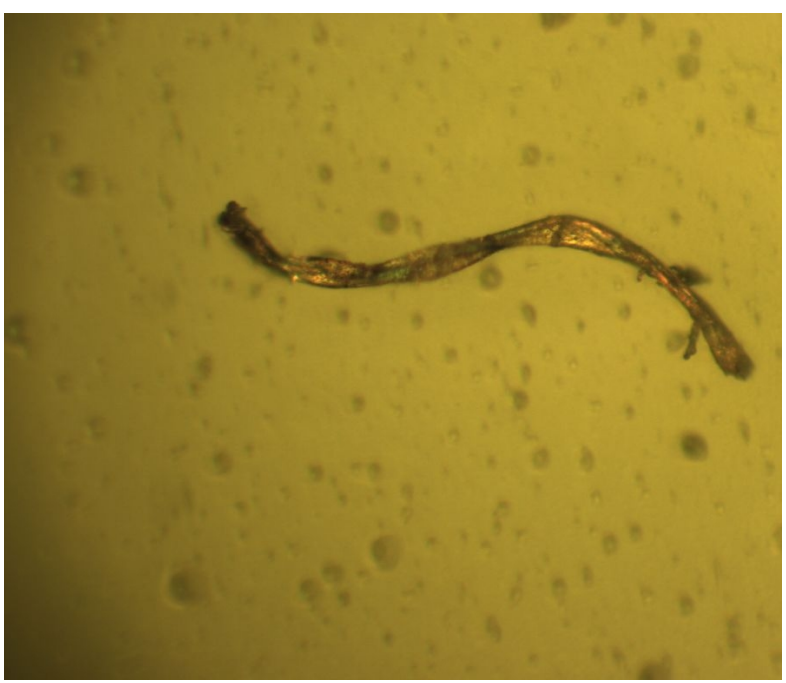

Fig.7 Spiral cutting shape particles

The worn pellets shown in figure. 6 and figure. 7 are tenuous, such as the cutting of the wear debris, which is typical characteristic of the cutting wear debris. The worn pellets shown in figure 6 , which length is about $37.6 \mu \mathrm{m}$, has no spiral. But the worn pellets shown in figure.7, which length is about $42 \mu \mathrm{m}$, has obvious spiral. We could get the conclusion preliminary that these two kinds of worn pellets is the product of the abrasive wear in different friction pair. 

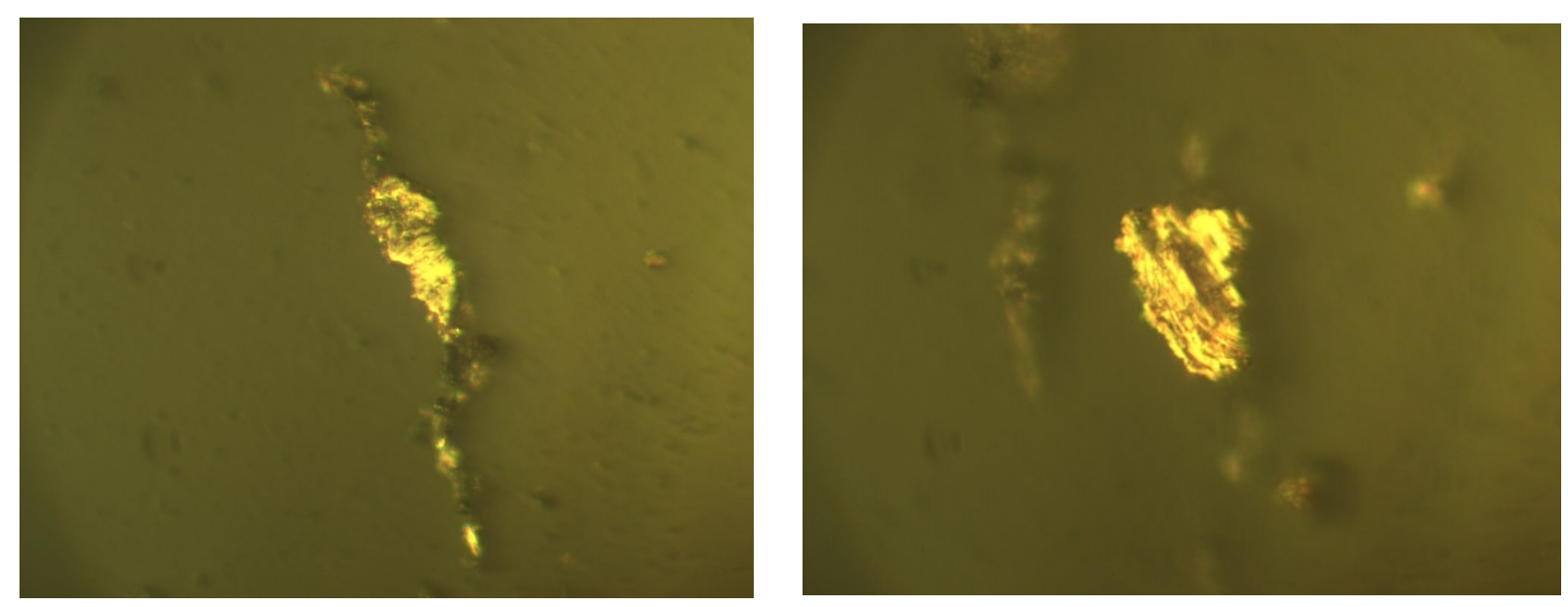

Figure.8 Irregular particles

Pellets in figure. 8 have an irregular shape with coarse surface and parallel scratches which are typical serious slipping wear, belonging to adhesive wear, but pellets have no blue heat tint. From those we can conclude the machine is in the prelimary stage of adhesive wear.

According to the above analysis we have the following findings after disport the machine :

There is slight scuffing phenomenon.

There are scratches in the surface of cylinder sleeves and the journal of crankshaft.

The drive gear has been worn.

These facts above are all the mechanization analysis by testing oil.

\section{Conclusions}

It's practical to analyze the wearing condition of Cummins marine diesel engine by using ferrographie oil analysis technology.

By adopt image analysis system we judge the wearing condition of oil sample.

By collecting oil sample depending on different situation, we can learn the tendency of wearing by analyzing the working condition of Cummins marine diesel engine, which will provide on-condition maintenance with evidence.

\section{References}

[1] Wenshi Zhu, Huang Ping, Principles of Tribology, Tsinghua university press, Beijing, 1990. In Chinese.

[2] Haijun Wei, Peiting Sun, Transactions of Csice, J. 23(2005)89-91. In Chinese.

[3] Zhuguo Li, Shiming Wei, Chinese Internal Combustion Engine Engineering, J. 28(2001)78-83. In Chinese.

[4] Zhuguo Li, Oil Analysis Diagnosis Technology, Shanghai science and technology press, Shanghai, 1997. In Chinese.

[5] Qiming Yang, Wear Particle Analysis-Grinding Grain of Atlas and Iron Spectrum Technology, China railway publishing house, Beijing, 2002. In Chinese 